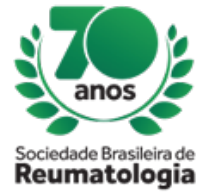

\title{
LUPUS MYOCARDITIS DIAGNOSED BY 18-FDG PETSCAN
}

Bruno Bordallo Corrêa (UFRJ, RJ, RJ, Brasil), Julio Cesár da Silva Borges (UFRJ, Rio de Janeiro, RJ, Brasil), Mirhelen Mendes de Abreu (UFRJ, Rio de Janeiro, RJ, Brasil), Vivian Mabel Orsi Dorado (UFRJ, Rio de Janeiro, RJ, Brasil), Hector Fabian Bernal (UFRJ, Rio de Janeiro, RJ, Brasil), Hany Kelly Araujo Cruz (UFRJ, Rio de Janeiro, RJ, Brasil), Lizeth Chaparro del Portilho (UFRJ, Rio de Janeiro, RJ, Brasil), Julia Yoneshigue Laranja de Oliveira (UFRJ, Rio de Janeiro, RJ, Brasil), Blanca Elena Rios Gomes Bica (UFRJ, Rio de Janeiro, RJ, Brasil)

\section{BACKGROUND}

Cardiovascular disease and heart failure (HF) are important causes of death in patients with systemic lupus erythematosus (SLE). Mechanisms that trigger increased HF and cardiovascular disease incidence in SLE patients are still not well understood, but myocardial inflammation and lupus myocarditis seem to play an important role in the pathophysiology of the disease. Myocarditis associated with SLE presents a prevalence of $57 \%$ of endomyocardial biopsies in postmortem studies, but is still underdiagnosed in SLE patients due to the non-specific nature of the symptoms.

\section{CASE REPORT}

A 66 year old female patient with SLE, rheumatoid arthritis, pulmonary interstitial disease, using Abatacept and prednisone, hydroxychloroquine had been discontinued due to maculopathy. There was also a double valve lesion with stenosis and severe aortic valve regurgitation and moderate mitral and tricuspid regurgitation, with no defined etiology, but with a left ventricular ejection fraction preserved in echocardiogram of 2007. She presented an episode of dyspnea on the efforts in 2015 and the possibility was raised of myocarditis or evolution of the orovalvar lesion being submitted to corticoid treatment and evolving with clinical improvement. The patient was admitted to the emergency with progressive dyspnea at rest, tachycardia and tachypnea started about 2 months ago and an episode of syncope on 06/03/2019 undergoing a new echocardiogram revealing LV dysfunction and diffuse hypokinesia. PET-scan was then requested to evaluate the possibility of myocarditis. Patient presented intense diffuse hypermetabolism in LV on PET-scan with 18-FDG demonstrating inflammatory myocardial activity compatible with myocarditis. (figure 1.)

\section{CONCLUSION}

The non-specificity of the symptoms, the subclinical character of the disease in some patients as well as the absence of a sensitive and specifc method for diagnoses contributes to the underdiagnosis of lupus myocarditis. The use of PET-scan with 18-FDG seems to be a promising tool in the diagnosis of this disease allowing its adequate treatment and possibly reducing its morbidity. 